\title{
Bounds on moments of the energy spectrum for weak solutions of the three-dimensional Navier-Stokes equations
}

\author{
Charles R. Doering* \\ Department of Mathematics, University of Michigan, Ann Arbor, Michigan, \\ MI 48109-1109, USA
}

J. D. Gibbon

Department of Mathematics, Imperial College of Science, Technology $\mathcal{E}$ Medicine, London $S W 7$ 2BZ, UK

\begin{abstract}
We consider weak solutions of the incompressible Navier-Stokes equations in a finite three-dimensional volume without boundaries. For flows driven by a time independent body-force, we focus on some long-time averaged ratios of norms of derivatives of the velocity field corresponding to moments of the Fourier energy spectrum of the solutions. The rigorous bounds derived here - without making any additional assumptions on regularity of solutions - are consistent with an asymptotic $k^{-8 / 3}$ energy spectrum. In contrast, if spatial fluctuations in velocity gradients are suppressed by assumption in the analysis, then the bounds are consistent with the $k^{-5 / 3}$ turbulent Kolmogorov energy spectrum. These results are interpreted and discussed in the context of spatial intermittency and the energy cascade picture of turbulent dynamics.
\end{abstract}

Key words: Navier-Stokes, weak solutions, rigorous bounds, Fourier energy spectrum, spatial intermittency, energy cascade

PACS: 47.10.+g, 47.27.Jv, 47.53.+n, 02.30.Jr

* Corresponding author.

Email addresses: doering@umich.edu (Charles R. Doering), j.d.gibbon@ic.ac.uk (J. D. Gibbon). 


\section{Introduction}

The mathematical analysis of the Navier-Stokes equations stands as one of the premier challenges for the 21st century. While the open question of regularity of solutions holds a prominent place [1-5], there is also an active line of investigation that concentrates on the extraction of general and physically relevant estimates for weak solutions. Sharp bounds on quantities such as the time-averaged energy dissipation rate make contact with notions from turbulence theory and are directly relevant, in a number of cases, to both computational and real physical experiments on turbulent flows $[6,7]$. In this paper we study some time-averaged moments of the Fourier energy spectrum of weak solutions of the incompressible three-dimensional Navier-Stokes equations for flows driven by a narrow-band time-independent body force in the absence of boundaries. We interpret the results presented here in the context of the structure of the spectrum for turbulent flows, even though they apply more generally than this, because the energy spectrum plays such a central role in turbulence theory.

The specific aim of this work is to derive a priori upper bounds on quantities of the type

$$
\left\langle\tilde{\kappa}_{n}\right\rangle \equiv\left\langle\left(\frac{\left\|\nabla^{n} \boldsymbol{u}\right\|_{2}}{\|\boldsymbol{u}\|_{2}}\right)^{1 / n}\right\rangle
$$

in terms of the length scale $L$ in the flow domain, the length scale $\ell$ in the applied body force, and the Reynolds number $R e=U \ell / \nu$, where $U$ is the timeaveraged root-mean-square velocity and $\nu$ the kinematic viscosity. In (1), $\boldsymbol{u}$ is a weak solution of the Navier-Stokes equations, $\langle\cdot\rangle$ denotes the long time average, and the notation for norms $\|\boldsymbol{u}\|_{2}^{2}=\int_{V}|\boldsymbol{u}|^{2} d V$ displayed in (1) has been adopted. The time-averaged "wavenumbers" $\left\langle\tilde{\kappa}_{n}\right\rangle$ are of interest precisely because they are moments of the Fourier energy spectrum of the solution. They could be finite even for weak solutions, so the estimates derived here do not shed any new light on the regularity issue. For technical reasons we will employ slightly modified time-averaged ratios of norms $\left\langle\kappa_{n}\right\rangle$, corresponding to the same moments as in (1), but which also take account of the body forces driving the fluid. We find that in the asymptotic limit $\nu \rightarrow 0$, with all other parameters held fixed,

$$
\ell\left\langle\kappa_{n}\right\rangle \leq c_{n}\left(\frac{L}{\ell}\right)^{\frac{3(n-1)}{n}} R e^{3-\frac{5}{2 n}+\frac{\delta}{n}} .
$$

The parameter $\delta$ is arbitrarily small and lies in the range $0<\delta<1 / 2$ and the prefactors $c_{n}$ are absolute constants that do not depend on any of the 
parameters of the problem, including $\delta$.

The scaling in these upper bounds may be interpreted in terms of the structure of the Fourier spectrum $\mathcal{E}(k)$ if we assume a scaling form $\mathcal{E} \sim k^{-q}$ up to the cut-off wavenumber $\ell k_{c} \sim R e^{q_{c}}$. Disregarding the "correction" from the arbitrarily small value of $\delta$, the a priori bounds in (2) are consistent with $q=8 / 3$ and $q_{c}=3$. Such a $k^{-8 / 3}$ spectrum has arisen in at least two previous studies. Sulem and Frisch [8] have shown that a $k^{-8 / 3}$ spectrum is the borderline steepness capable of sustaining an energy cascade in the Navier-Stokes equations when the total energy is finite. Mandelbrot [9], and later Frisch, Sulem and Nelkin in their toy $\beta$-model $[10,11]$, came upon this same scaling exponent as an extreme limit of intermittency in the energy cascade. They found that if the energy dissipation is assumed to be concentrated on a fractal set (in space) of dimension $D=8-3 q$, then the energy spectrum scaling is of the form $\mathcal{E} \sim k^{-q}$. Within this picture, the exponent $q=8 / 3$ thus corresponds to dissipation concentrated at zero-dimensional points in space. Interestingly, the conventional Kolmogorov $k^{-5 / 3}$ spectrum for homogeneous isotropic turbulence is associated with $D=3$. That is, a complete lack of intermittency with dissipation spread uniformly in space is consistent with $q=5 / 3$. As will be discussed further in the concluding section of this paper, if we complement our analysis with the additional assumption that the sup-norm of the velocity derivative scales in magnitude like its root-mean-square at high $R e$ (that is, if $\|\nabla \boldsymbol{u}\|_{\infty} \sim L^{-3 / 2}\|\nabla \boldsymbol{u}\|_{2}$ as $R e \rightarrow \infty$ ) then we recover the scaling bounds

$$
\ell\left\langle\kappa_{n}\right\rangle \leq c_{n} R e^{\frac{3}{4}-\frac{1}{4 n}+\frac{\delta}{n}}
$$

These exponents are consistent with the exponents $q=5 / 3$ and $q_{c}=3 / 4$ with $k_{c}$ corresponding to the Kolmogorov dissipation wavenumber. Thus we find that the technical assumption of uniform dissipation expressed in the form above implies that Kolmogorov's scaling exponents are consistent with this extreme limit for weak solutions of the Navier-Stokes equations.

Moreover, our result can (and will) be generalized to make the following observation: if we assume that intermittency fluctuations are controlled so that for some $2 \leq m \leq \infty,\|\nabla \boldsymbol{u}\|_{\infty} \sim L^{-3 / m}\|\nabla \boldsymbol{u}\|_{m}$ as $R e \rightarrow \infty$, then

$$
\ell\left\langle\kappa_{n}\right\rangle \leq c\left(\frac{L}{\ell}\right)^{\frac{(3 q-5)(n-1)}{n(9-3 q)}} R e^{\frac{1}{3-q}-\frac{1}{2 n}\left(\frac{q-1}{3-q}\right)+\frac{\delta}{n}}
$$

where $q=\frac{8}{3}-\frac{2}{m}$. As $m$ ranges between 2 and $\infty$, the corresponding value of $q$ ranges from the Kolmogorov $5 / 3$ to the $8 / 3$ limit. In terms of the dimension of the set upon which the dissipation concentrates in the context of the $\beta$-model, this result corresponds to $D=6 / m$. 
An alternative articulation of the implications of the upper bounds on the moments in (1) is that we can rule out certain scaling hypotheses for the energy spectrum. Indeed, the bounds on $\left\langle\kappa_{n}\right\rangle$ imply that the assumption of an energy spectrum of the form $\mathcal{E} \sim k^{-q}$ (up to cut-off) with an energy dissipation rate $\epsilon>0$ asymptotically independent of $\nu$ as $\nu \rightarrow 0$, is inconsistent with any value of $q$ satisfying $8 / 3<q<3$.

The rest of this paper is organized as follows. In Section 2, we present the system description for the flows we will study, including all the relevant definitions. Section 3 contains the proof of the central result, which proceeds in three main steps. The concluding Section 4 is a summary and further discussion of the results, including a more detailed presentation of the effect of the assumption of lack of intermittency in the spatial distribution of the energy dissipation.

\section{System description and definitions}

We consider the Navier-Stokes equations on a periodic cube $V=[0, L]^{3}$

$$
\begin{aligned}
& \boldsymbol{u}_{t}+\boldsymbol{u} \cdot \nabla \boldsymbol{u}=\nu \Delta \boldsymbol{u}-\nabla p+\boldsymbol{f}(\boldsymbol{x}), \\
& \nabla \cdot \boldsymbol{u}=0
\end{aligned}
$$

where $\nu$ is the kinematic viscosity, $p$ is the pressure and the applied body force $\boldsymbol{f}(\boldsymbol{x})$ is mean-zero and divergence-free. Hence, without loss of generality, we may take the solution $\boldsymbol{u}(\boldsymbol{x}, t)$ to be mean-zero at all times. For simplicity we will consider narrow-band body forces with a single length scale $\ell$; that is, with Fourier components only at wavenumber $k=\ell^{-1}$. Note that $\ell \leq L / 2 \pi$. For finite energy initial data the Navier-Stokes equations admit weak solutions in $L^{2}(V)$ at each instant of time, with finite time integrals of the $L^{2}$-norms of the velocity gradients $[1-5]$.

We measure the intensity of the driving force by the Grashof number

$$
G r=\frac{f \ell^{3}}{\nu^{2}}
$$

where $f^{2}=L^{-3}\|\boldsymbol{f}\|_{2}^{2}$. With our assumption of narrow-band forcing, norms of gradients of $\boldsymbol{f}(\boldsymbol{x})$ are related to the norm of $\boldsymbol{f}(\boldsymbol{x})$ itself via

$$
\|\boldsymbol{f}\|_{2}^{2}=\ell^{2 n}\left\|\nabla^{n} \boldsymbol{f}\right\|_{2}^{2}
$$


We will focus on long time averaged quantities using the notation

$$
\langle\Phi(\cdot)\rangle=\operatorname{LiM}_{t \rightarrow \infty}\left(\frac{1}{t} \int_{0}^{t} \Phi(s) d s\right)
$$

where LIM is a generalized long-time limit for functionals of (weak) statistical solutions of the Navier-Stokes equations [4,12-14]. The root mean square velocity scale is $U$ and is defined by

$$
U^{2}=L^{-3}\left\langle\|\boldsymbol{u}\|_{2}^{2}\right\rangle
$$

and the Reynolds number by

$$
R e=\frac{U \ell}{\nu}
$$

The time-averaged energy dissipation rate (an a priori finite quantity for weak solutions) is defined as

$$
\epsilon=\nu L^{-3}\left\langle\|\nabla \boldsymbol{u}\|_{2}^{2}\right\rangle
$$

With these definitions in hand we can identify some of the conventional small length scales of turbulence theory: the Taylor micro-scale is

$$
\lambda_{T}=\left(\frac{\nu U^{2}}{\epsilon}\right)^{1 / 2}
$$

and the Kolmogorov length $\lambda_{K}$ is

$$
\lambda_{K}=\left(\frac{\nu^{3}}{\epsilon}\right)^{1 / 4}
$$

There are a number of known relationships among the quantities valid for weak solutions within the specific system considered here. For example, the energy dissipation rate can be estimated in terms of the root mean square velocity scale [7] by

$$
\epsilon \leq c_{1} \nu \frac{U^{2}}{\ell^{2}}+c_{2} \frac{U^{3}}{\ell}=\nu^{3} \ell^{-4}\left(c_{1} R e^{2}+c_{2} R e^{3}\right)
$$


where $c_{1}$ and $c_{2}$ are absolute constants. This fact can be recast as lower bounds on the Taylor and Kolmogorov scales

$$
\lambda_{T} \geq \ell\left(c_{1}+c_{2} R e\right)^{-1 / 2} \sim c \ell R e^{-1 / 2} \quad \text { as } R e \rightarrow \infty
$$

and

$$
\lambda_{K} \geq \ell\left(c_{1} R e^{2}+c_{2} R e^{3}\right)^{-1 / 4} \sim c \ell R e^{-3 / 4} \quad \text { as } R e \rightarrow \infty .
$$

For the problem formulated here, the Grashof number $G r$, is the natural control parameter, not the Reynolds number Re. We may, however, be assured of achieving high Reynolds number solutions if the Grashof number is sufficiently high. Indeed, Doering and Foias [7] have proved that for body-forced flows such as these

$$
R e \geq c G r^{1 / 2} \quad \text { as } \quad G r \rightarrow \infty .
$$

Moreover, there is an explicit lower bound on the energy dissipation rate at high $G r$ given by

$$
\epsilon \geq c \frac{\nu f}{L}=c \frac{\nu^{3}}{\ell^{3} L} G r \quad \text { as } \quad G r \rightarrow \infty .
$$

This result, although not appearing explicitly in this form in [7], follows in a straightforward way from the methods used in that paper.

Now we define the time dependent quantities

$$
H_{n}(t)=\left\|\nabla^{n} \boldsymbol{u}(\cdot, t)\right\|_{2}^{2}=\sum_{\boldsymbol{k}} k^{2 n}|\hat{\boldsymbol{u}}(\boldsymbol{k}, t)|^{2}
$$

where the Fourier transform is

$$
\hat{\boldsymbol{u}}(\boldsymbol{k}, t)=L^{-3} \int_{V} \exp (-i \boldsymbol{k} \cdot \boldsymbol{x}) \boldsymbol{u}(\boldsymbol{x}, t) d V .
$$

The (instantaneous) $2 n$-th moment of the Fourier energy spectrum is

$$
\frac{H_{n}(t)}{H_{0}(t)}=\frac{\left\|\nabla^{n} \boldsymbol{u}\right\|_{2}^{2}}{\|\boldsymbol{u}\|_{2}^{2}}=\frac{\sum_{\boldsymbol{k}} k^{2 n}|\hat{\boldsymbol{u}}(\boldsymbol{k}, t)|^{2}}{\sum_{\boldsymbol{k}}|\hat{\boldsymbol{u}}(\boldsymbol{k}, t)|^{2}} \equiv \tilde{\kappa}(t)^{2 n}
$$

It is not known if the wavenumber moments remain finite for all times for $n \geq 1$; this is the essence of the regularity problem. But some time averages 
of the $\tilde{\kappa}_{n}(t)$ may nevertheless be finite even for weak solutions and it is these that attract our attention here.

For technical reasons in the proof in the next section, we must address the possibility of small values of the quantities $H_{n}$ in order to circumvent problems that may arise when dividing by these (squared) semi-norms. Hence we introduce the slightly modified quantities

$$
F_{n}=H_{n}+\tau^{2}\left\|\nabla^{n} \boldsymbol{f}\right\|_{2}^{2}
$$

where the "time-scale" $\tau$ will be chosen for our convenience. So long as $\tau \neq 0$, the $F_{n}$ are bounded away from zero by the explicit value $\tau^{2} L^{3} \ell^{-2 n} f^{2}$. Moreover, we may choose $\tau$ to depend on the parameters of the problem such that $\left\langle F_{n}\right\rangle \sim$ $\left\langle H_{n}\right\rangle$ as $G r \rightarrow \infty$. To see how to achieve this, let us define

$$
\tau=\ell^{2} \nu^{-1} G r^{-(\delta+1 / 2)}
$$

with $\delta>0$, which is a parameter yet to be determined. Then the additional term in $(23)$ is

$$
\begin{aligned}
\tau^{2}\left\|\nabla^{n} \boldsymbol{f}\right\|_{2}^{2} & =L^{3} \nu^{-2} \ell^{4-2 n} f^{2} G r^{-(2 \delta+1)} \\
& =\nu^{2} \ell^{-(2 n+2)} L^{3} G r^{1-2 \delta}
\end{aligned}
$$

Recalling the a priori bound on the far right hand side of (19)

$$
\begin{aligned}
\tau^{2}\left\|\nabla^{n} \boldsymbol{f}\right\|_{2}^{2} & \leq c \epsilon \ell^{-(2 n-1)} L^{4} \nu^{-1} G r^{-2 \delta} \\
& =c\left(\frac{L}{\ell}\right)^{(2 n-1)} L^{-2(n-1)}\left\langle H_{1}\right\rangle G r^{-2 \delta} \\
& \leq c\left(\frac{L}{\ell}\right)^{(2 n-1)}\left\langle H_{n}\right\rangle G r^{-2 \delta}
\end{aligned}
$$

where we have used Poincaré's inequality at the last step. Hence, for any $\delta>0$ we see that the additional forcing term in (23) becomes negligible with respect to $\left\langle H_{n}\right\rangle$ as $G r \rightarrow \infty$. We leave $\delta$ arbitrary otherwise at this stage, although we will restrict it further in the course of proving the two key Lemmas in the next section.

Given the definition of the $F_{n}$ in (23) and of $\tau$ in (24), we now define the family of time-dependent wavenumber magnitudes

$$
\kappa_{n, r}(t)=\left(\frac{F_{n}}{F_{r}}\right)^{\frac{1}{2(n-r)}} .
$$


For the case $r=0$ we will simply write $\kappa_{n}(t) \equiv \kappa_{n, 0}(t)$. These quantities are naturally ordered according to $\kappa_{n, r} \leq \kappa_{n+1, r}$ for $0 \leq r<n$ and $\kappa_{n, r} \leq \kappa_{n, r+1}$ for $1 \leq r+1<n$. These are the quantities whose time averages will be bounded in the next section. For the reader who wants to avoid the technical detail of the next section, we will prove in Lemma 1 that for $0<\delta<1 / 2$,

$$
\ell\left\langle\kappa_{n, r}\right\rangle \leq c_{n, r}\left(\frac{L}{\ell}\right)^{3} R e^{3}
$$

as $G r \rightarrow \infty$ for $1 \leq r<n$. This result is subsequently sharpened further in the case $r=0$, corresponding more directly to the moments of the energy spectrum. In Theorem 1 we will prove that for $0<\delta<1 / 2$ and $n \geq 2$

$$
\ell\left\langle\kappa_{n}\right\rangle=\ell\left\langle\kappa_{n, 0}\right\rangle \leq c_{n}\left(\frac{L}{\ell}\right)^{\frac{3(n-1)}{n}} R e^{3-\frac{5}{2 n}+\frac{\delta}{n}}
$$

as $G r \rightarrow \infty$.

\section{Bounds on $\left\langle\kappa_{n}\right\rangle$ and $\left\langle\kappa_{n, r}\right\rangle$}

The following Lemma concerns finding upper bounds on $\left\langle\kappa_{n, r}\right\rangle$ in terms of $R e$.

Lemma 1 For $1 \leq r<n$ and $0<\delta<1 / 2$

$$
\ell\left\langle\kappa_{n, r}\right\rangle \leq c_{n, r}\left(\frac{L}{\ell}\right)^{3} R e^{3}
$$

as $R e \rightarrow \infty$, where the coefficients $c_{n, r}$ do not depend on any parameters of the problem.

Proof: Let us focus upon $\left\langle\kappa_{n+1, n}\right\rangle$.

$$
\begin{aligned}
\left\langle\kappa_{n+1, n}\right\rangle=\left\langle\left(\frac{F_{n+1}}{F_{n}}\right)^{1 / 2}\right\rangle & =\left\langle\left(\frac{F_{n+1}}{F_{n}^{\frac{2 n}{2 n-1}}}\right)^{1 / 2} F_{n}^{\frac{1}{2(2 n-1)}}\right\rangle \\
& \leq\left\langle\frac{F_{n+1}}{F_{n}^{\frac{2 n}{2 n-1}}}\right\rangle^{1 / 2}\left\langle F_{n}^{\frac{1}{2 n-1}}\right\rangle^{1 / 2}
\end{aligned}
$$

The quantities $\left\langle F_{n}^{\frac{1}{2 n-1}}\right\rangle$ were first shown by Foias, Guillopé and Temam to be bounded [15]. Two things are now required: an estimate for $\left\langle F_{n}^{\frac{1}{2 n-1}}\right\rangle$ in terms 
of $\kappa_{n, n-1}$ and an estimate for the square rooted time averaged ratio on the right hand side of (31). One simple relation between them is

$$
\left\langle F_{n}^{\frac{1}{2 n-1}}\right\rangle^{2 n-1} \leq\left\langle\kappa_{n, r}\right\rangle^{2(n-r)}\left\langle F_{r}^{\frac{1}{2 r-1}}\right\rangle^{2 r-1}
$$

which comes about by re-arrangement and a Hölder inequality. For the first quantity we simply put $r=1$ in (32). Since $\kappa_{n, 1} \leq \kappa_{n, n-1}$ for $n \geq 2$, we have

$$
\left\langle F_{n}^{\frac{1}{2 n-1}}\right\rangle \leq\left\langle\kappa_{n, n-1}\right\rangle^{\frac{2(n-1)}{2 n-1}}\left\langle F_{1}\right\rangle^{\frac{1}{2 n-1}}
$$

which gives

$$
\left\langle\kappa_{n+1, n}\right\rangle^{2} \leq\left\langle\frac{F_{n+1}}{F_{n}^{\frac{2 n}{2 n-1}}}\right\rangle\left\langle\kappa_{n, n-1}\right\rangle^{\frac{2(n-1)}{2 n-1}}\left\langle F_{1}\right\rangle^{\frac{1}{2 n-1}} .
$$

To inject information from the Navier-Stokes equations into (34), it is now necessary to find an estimate for the time averaged ratio on the right hand side. We consider the ladder of differential inequalities satisfied by the $H_{n}$ derived in [5]

$$
\frac{1}{2} \dot{H}_{n} \leq-\frac{\nu}{2} H_{n+1}+c_{n} \nu^{-1}\|\boldsymbol{u}\|_{\infty}^{2} H_{n}+H_{n}^{1 / 2}\left\|\nabla^{n} \boldsymbol{f}\right\|_{2}
$$

(Note that in the calculations that follow we will manipulate the $H_{n}$ formally even though they are not known to be finite pointwise in time for weak solutions; the end results may be justified by proceeding from a Galerkin approximation to the solutions and then removing the regularization in the final results.) Having established that for $\delta>0$ the additive forcing part of $F_{n}$ is small under the stated conditions, we are ready to find how the $F_{n}$ evolve. Adding and subtracting the quantity $\frac{1}{2} \nu \tau^{2}\left\|\nabla^{n+1} \boldsymbol{f}\right\|_{2}^{2}$, we see that

$$
\frac{1}{2} \dot{F}_{n} \leq-\frac{\nu}{2} F_{n+1}+c_{n} \nu^{-1}\|\boldsymbol{u}\|_{\infty}^{2} F_{n}+H_{n}^{1 / 2}\left\|\nabla^{n} \boldsymbol{f}\right\|_{2}+\frac{1}{2} \nu \tau^{2}\left\|\nabla^{n+1} \boldsymbol{f}\right\|_{2}^{2} .
$$

Now break up the first of the last pair of terms by Young's inequality as follows way using $g \tau^{2}>0$ as a parameter, where $g$ is to be suitably chosen below.

$$
\begin{aligned}
H_{n}^{1 / 2}\left\|\nabla^{n} \boldsymbol{f}\right\|_{2}+\frac{1}{2} \nu \tau^{2}\left\|\nabla^{n+1} \boldsymbol{f}\right\|_{2}^{2} & \leq \frac{1}{2 g \tau^{2}} H_{n}+\frac{g \tau^{2}}{2}\left\|\nabla^{n} \boldsymbol{f}\right\|_{2}^{2}+\frac{\nu \tau^{2}}{2}\left\|\nabla^{n+1} \boldsymbol{f}\right\|_{2}^{2} \\
& \leq \frac{1}{2 g \tau^{2}} H_{n}+\frac{1}{2}\left(g+\frac{\nu}{\ell^{2}}\right) \tau^{2}\left\|\nabla^{n} \boldsymbol{f}\right\|_{2}^{2}
\end{aligned}
$$


where we have inserted the forcing length scale $\ell$ to reduce a derivative on $\boldsymbol{f}$. Now we pick the two coefficients of $H_{n}$ and $\tau^{2}\left\|\nabla^{n} \boldsymbol{f}\right\|_{2}^{2}$ to be equal, finding that

$$
g=-\frac{\nu}{2 \ell^{2}}+\left\{\frac{\nu^{2}}{4 \ell^{4}}+\frac{1}{\tau^{2}}\right\}^{1 / 2} .
$$

With $\tau$ chosen as in (24) with $\delta>0$,

$$
g=\tau^{-1}\left(\sqrt{1+\frac{1}{4 G r^{2 \delta+1}}}-\frac{1}{2 G r^{\delta+1 / 2}}\right) .
$$

Consequently, $g \sim \tau^{-1}$ as $G r \rightarrow \infty$. In this limit the ladder for $F_{n}$ can be written as

$$
\frac{1}{2} \dot{F}_{n} \leq-\frac{\nu}{2} F_{n+1}+\left(c_{n} \nu^{-1}\|\boldsymbol{u}\|_{\infty}^{2}+\frac{1}{2 g \tau^{2}}\right) F_{n} .
$$

Using Agmon's inequality,

$$
\|\boldsymbol{u}\|_{\infty} \leq c\left\|\nabla^{n} \boldsymbol{u}\right\|_{2}^{b}\|\nabla \boldsymbol{u}\|_{2}^{1-b}
$$

where, for $n>2, b=\frac{1}{2(n-1)}$, we obtain

$$
\|\boldsymbol{u}\|_{\infty} \leq c_{n} \kappa_{n, 1}^{1 / 2} F_{1}^{1 / 2}
$$

which gives

$$
\frac{1}{2} \dot{F}_{n} \leq-\frac{\nu}{2} F_{n+1}+\left(c_{n} \nu^{-1} \kappa_{n, 1} F_{1}+\frac{1}{2 g \tau^{2}}\right) F_{n} .
$$

Dividing by $F_{n}^{\frac{2 n}{2 n-1}}$ and time averaging we see that for $n \geq 2$

$$
\begin{aligned}
\left\langle\frac{F_{n+1}}{F_{n}^{\frac{2 n}{2 n-1}}}\right\rangle & \leq c \nu^{-2}\left\langle\kappa_{n, 1} F_{1} F_{n}^{1-\frac{2 n}{2 n-1}}\right\rangle+\frac{1}{\nu g \tau^{2}}\left\langle F_{n}^{-\frac{1}{2 n-1}}\right\rangle \\
& =c \nu^{-2}\left\langle\kappa_{n, 1}^{\frac{1}{2 n-1}} F_{1}^{\frac{2(n-1)}{2 n-1}}\right\rangle+\frac{1}{\nu g \tau^{2}}\left\langle F_{n}^{-\frac{1}{2 n-1}}\right\rangle .
\end{aligned}
$$

Because $\left\|\nabla^{n} \boldsymbol{f}\right\|_{2}^{2}=\ell^{-2 n}\|\boldsymbol{f}\|_{2}^{2}$,

$$
F_{n} \geq \tau^{2} \ell^{-2 n} L^{3} f^{2}
$$


and using the fact that $\kappa_{n, 1} \leq \kappa_{n, n-1}$ and $g \sim \tau^{-1}$ for $G r \rightarrow \infty$, (44) becomes

$$
\left\langle\frac{F_{n+1}}{F_{n}^{\frac{2 n}{2 n-1}}}\right\rangle \leq c \nu^{-2}\left\langle\kappa_{n, n-1}\right\rangle^{\frac{1}{2 n-1}}\left\langle F_{1}\right\rangle^{\frac{2(n-1)}{2 n-1}}+K
$$

where

$$
K=\frac{1}{\nu \tau}\left(\frac{\ell^{2 n}}{\tau^{2} L^{3} f^{2}}\right)^{\frac{1}{2 n-1}}=\frac{1}{\nu \tau}\left(\frac{\ell^{2 n+6}}{\tau^{2} L^{3} \nu^{4} G r^{2}}\right)^{\frac{1}{2 n-1}} .
$$

The estimate (46) now needs to be substituted into (34), which yields

$$
\left\langle\kappa_{n+1, n}\right\rangle^{2} \leq c_{n} \nu^{-2}\left\langle\kappa_{n, n-1}\right\rangle\left\langle F_{1}\right\rangle+K\left\langle\kappa_{n, n-1}\right\rangle^{\frac{2(n-1)}{2 n-1}}\left\langle F_{1}\right\rangle^{\frac{1}{2 n-1}}
$$

Using the fact that $\left\langle\kappa_{n, n-1}\right\rangle \leq\left\langle\kappa_{n+1, n}\right\rangle$ and dividing by $\left\langle\kappa_{n, n-1}\right\rangle^{\frac{2(n-1)}{2 n-1}}$, a Hölder inequality gives

$$
\left\langle\kappa_{n, n-1}\right\rangle^{\frac{2 n}{2 n-1}} \leq \underbrace{c_{n}\left(\nu^{-2}\left\langle F_{1}\right\rangle\right)^{\frac{2 n}{2 n-1}}}_{\text {1st term }}+\underbrace{\left(\frac{2 n}{2 n-1}\right) K\left\langle F_{1}\right\rangle^{\frac{1}{2 n-1}}}_{\text {2nd term }} .
$$

The task now is to compare the magnitudes of the first and second terms on the right hand side of (49). Ignoring constants,

$$
\frac{2 \text { nd term }}{1 \text { st term }}=\frac{K}{\nu^{-\frac{4 n}{2 n-1}}\left\langle F_{1}\right\rangle} \text {. }
$$

Using the fact that the energy dissipation rate $\epsilon$ is bounded both from above and below, the latter being given in (19)

$$
c \frac{\nu^{3}}{\ell^{3} L} G r \leq \epsilon \leq \nu L^{-3}\left\langle F_{1}\right\rangle
$$

we find that

$$
\frac{2 \text { nd term }}{1 \text { st term }} \leq\left(\frac{\ell}{L}\right)^{\frac{4 n+1}{2 n-1}} G r^{\left(\delta-\frac{1}{2}\right)\left(\frac{2 n+1}{2 n-1}\right)}
$$

Hence we see that the choice of $\delta$ in the range $0<\delta<1 / 2$ renders the 2nd term negligible compared to the first for high $G r$. Thus for $n \geq 2$ with $1 \leq r \leq n-1$ we have, as $G r \rightarrow \infty$,

$$
\left\langle\kappa_{n, r}\right\rangle \leq\left\langle\kappa_{n, n-1}\right\rangle \leq c_{n} \nu^{-2}\left\langle F_{1}\right\rangle \text {. }
$$


Now we know that $\left\langle F_{1}\right\rangle \sim\left\langle H_{1}\right\rangle$ for $G r \rightarrow \infty$ as shown in (26). Hence we need only consider the first term in (49) to get

$$
\left\langle\kappa_{n, r}\right\rangle \leq c_{n} L^{3} \nu^{-3} \epsilon \leq c_{n} L^{3} \ell^{-4} R e^{3}
$$

as $G r \rightarrow \infty$. Finally, we note that the result of the theorem in (30), applied to (33), gives upper bounds on the quantities first considered by Foias, Guillopé and Temam [15].

Next we prove a short Lemma that provides the other essential element for the final result.

Lemma 2 There is an absolute constant c such that as $G r \rightarrow \infty$

$$
\left\langle\kappa_{1}^{2}\right\rangle \equiv\left\langle\kappa_{1,0}^{2}\right\rangle \leq c \ell^{-2} R e^{1+2 \delta} .
$$

Proof: The energy evolution equation for weak solutions of the Navier-Stokes equations is

$$
\frac{1}{2} \dot{H}_{0} \leq-\nu H_{1}+\int_{V} \boldsymbol{u} \cdot \boldsymbol{f} d V
$$

(the inequality is necessary for weak solutions). Addition and subtraction of $\nu \tau^{2} \ell^{-2} L^{3} f^{2}$ to the right hand side, together with an application of the CauchySchwarz inequality in the last term, yields

$$
\frac{1}{2} \dot{F}_{0} \leq-\nu F_{1}+\|\boldsymbol{u}\|_{2} L^{3 / 2} f+\nu \tau^{2} \ell^{-2} L^{3} f^{2}
$$

Introducing a parameter $h>0$ in breaking up the middle term on the right hand side by Young's inequality leads to

$$
\frac{1}{2} \dot{F}_{0} \leq-\nu F_{1}+\frac{1}{2 h \tau^{2}}\|\boldsymbol{u}\|_{2}^{2}+\left(\frac{h}{2}+\frac{\nu}{\ell^{2}}\right) \tau^{2} L^{3} f^{2}
$$

Then $h$ is chosen as

$$
h=\tau^{-1}\left(\sqrt{1+\frac{1}{G r^{2 \delta+1}}}-\frac{1}{G r^{\delta+1 / 2}}\right)
$$


to equalize the coefficients of $\|\boldsymbol{u}\|_{2}^{2}$ and $\tau^{2} L^{3} f^{2}$, in which case $h \sim \tau^{-1}$ as $G r \rightarrow \infty$. We have

$$
\frac{1}{2} \dot{F}_{0}=-\nu F_{1}+c \tau^{-1} F_{0}
$$

Dividing each term by $F_{0}$ and time averaging, we find

$$
\nu\left\langle\kappa_{1,0}^{2}\right\rangle \leq c \tau^{-1}=c \nu \ell^{2} G r^{\delta+1 / 2} .
$$

Recalling the previously established fact that $R e \geq c G r^{1 / 2}$ as $G r \rightarrow \infty$ [7], the result follows immediately.

Finally we are in a position to prove the central point of these analyses:

Theorem 1 For $n \geq 2$ and $r=0$, and for any value of the parameter $\delta$ lying in the range $0<\delta<1 / 2$,

$$
\ell\left\langle\kappa_{n}\right\rangle \equiv \ell\left\langle\kappa_{n, 0}\right\rangle \leq c_{n}\left(\frac{L}{\ell}\right)^{\frac{3(n-1)}{n}} R e^{3-\frac{5}{2 n}+\frac{\delta}{n}}
$$

as $G r \rightarrow \infty$, where the constants $c_{n}$ do not depend on any system parameter (including $\delta$ ).

Proof: Consider the quantity

$$
\begin{aligned}
\left\langle\kappa_{n, 0}^{\frac{2 n}{2 n-1}}\right\rangle=\left\langle\left(\frac{F_{n}}{F_{0}}\right)^{\frac{1}{2 n-1}}\right\rangle & =\left\langle\kappa_{n, 1}^{\frac{2(n-1)}{2 n-1}}\left(\kappa_{1,0}^{2}\right)^{\frac{1}{2 n-1}}\right\rangle \\
& \leq\left\langle\kappa_{n, 1}\right\rangle^{\frac{2(n-1)}{2 n-1}}\left\langle\kappa_{1,0}^{2}\right\rangle^{\frac{1}{2 n-1}}
\end{aligned}
$$

Now

$$
\left\langle\kappa_{n, 0}\right\rangle \leq\left\langle\kappa_{n, 0}^{\frac{2 n}{2 n-1}}\right\rangle^{\frac{2 n-1}{2 n}} \leq\left\langle\kappa_{n, 1}\right\rangle^{\frac{(n-1)}{n}}\left\langle\kappa_{n, 0}^{2}\right\rangle^{\frac{1}{2 n}}
$$

and inserting the estimates for $\left\langle\kappa_{n, 1}\right\rangle$ from Lemma 1 and that for $\left\langle\kappa_{1,0}^{2}\right\rangle$ from Lemma 2, the proof is complete.

\section{Summary and discussion}

To summarize, we have shown that for weak solutions of the three-dimensional Navier-Stokes equations, at high values of the Grashof number, the time- 
averaged "wavenumbers" satisfy

$$
\ell\left\langle\kappa_{n}\right\rangle=\ell\left\langle\left(\frac{\left\|\nabla^{n} \boldsymbol{u}\right\|_{2}^{2}+\tau^{2}\left\|\nabla^{n} \boldsymbol{f}\right\|_{2}^{2}}{\|\boldsymbol{u}\|_{2}^{2}+\tau^{2}\|\boldsymbol{f}\|_{2}^{2}}\right)^{1 / 2 n}\right\rangle \leq c_{n}\left(\frac{L}{\ell}\right)^{\frac{3(n-1)}{n}} R e^{3-\frac{5}{2 n}+\frac{\delta}{n}}
$$

where $\delta$ is any number in range $0<\delta<1 / 2$. In the above, the prefactors $c_{n}$ are absolute constants. Although $\delta$ may be chosen arbitrarily small and positive, its distance from zero controls the rate, as $G r$ and $R e \rightarrow \infty$, at which the wavenumbers $\left\langle\kappa_{n}\right\rangle$ defined above may practically be identified with the moments of the Fourier power spectrum

$$
\left\langle\tilde{\kappa}_{n}\right\rangle \equiv\left\langle\left(\frac{\left\|\nabla^{n} \boldsymbol{u}\right\|_{2}}{\|\boldsymbol{u}\|_{2}}\right)^{1 / n}\right\rangle
$$

We have not proved that the $\left\langle\kappa_{n}\right\rangle$ directly control the $\left\langle\tilde{\kappa}_{n}\right\rangle$. Rather, in (26) we have been able to show that there is a correspondence between them in the sense that the mean values of the numerators and denominators of the $\left\langle\kappa_{n}\right\rangle$ converge to those of $\left\langle\tilde{\kappa}_{n}\right\rangle$ as a power of $G r$ as $G r \rightarrow \infty$ for any $\delta>0$. The smaller the value of $\delta$, the higher $G r$ may have to be in order for us to justify this identification of $\left\langle\kappa_{n}\right\rangle$ with $\left\langle\tilde{\kappa}_{n}\right\rangle$.

In fact the $\delta / n$ term in the Reynolds number exponent in (65) may be elimated altogether and replaced by some milder correction to scaling by the following device. The time scale $\tau$ defined in (24) was chosen to serve two purposes. One was to ensure that at high $G r$ we could justifiably neglect the additions to the numerators and denominators in the ratios defining the wavenumbers - at least we may neglect the additions for the purposes of discussion and interpretation. The other was to enable the technical step of dropping the second term in (49) in the proof of Lemma 1. In both of these situations the algebraic dependence of $\tau$ on $G r$ is not strictly necessary. For example, suppose we choose

$$
\tau=\nu^{-1} \ell^{2} G r^{-1 / 2} \psi(G r)
$$

where $\psi(x)$ is any real, positive, monotonically decreasing function on $[0, \infty)$ that satisfies

$$
\lim _{x \rightarrow \infty} \psi(x) \rightarrow 0 \quad \text { and } \quad \lim _{x \rightarrow \infty} x^{1 / 2} \psi(x) \rightarrow \infty .
$$

Then $\tau$ would still have served the purpose of ensuring that

$$
\frac{\tau^{2}\left\|\nabla^{n} \boldsymbol{f}\right\|_{2}^{2}}{\left\langle\left\|\nabla^{n} \boldsymbol{u}\right\|_{2}^{2}\right\rangle} \rightarrow 0 \quad \text { as } \quad G r \rightarrow \infty
$$


and the theorem would simply be

$$
\ell\left\langle\kappa_{n}\right\rangle \leq c_{n}\left(\frac{L}{\ell}\right)^{\frac{3(n-1)}{n}} R e^{3-\frac{5}{2 n}}\left[\psi\left(c R e^{2}\right)\right]^{-\frac{1}{2 n}} .
$$

So the asymptotic correction to scaling is effectively illusory. An example would be a function such as $\psi(x) \sim[\log (1+x)]^{-1}$ that produces only a logarithmic modification to the asymptotic scaling.

That being said, let us consider a physical interpretation of the $R e^{3-5 / 2 n}$ wavenumber scaling in the bounds in the context of statistical turbulence theory, restricting attention to forcing at the longest wavelength so that $\ell=L / 2 \pi$. Suppose that $G r$ is high enough and the resulting flow is turbulent, ergodic and isotropic enough that the wavenumbers $\left\langle\kappa_{n}\right\rangle$ may be identified with the moments of the energy spectrum $\mathcal{E}(k)$ according to

$$
\left\langle\kappa_{n}\right\rangle^{2 n} \approx \frac{\int_{\ell^{-1}}^{\infty} k^{2 n} \mathcal{E}(k) d k}{\int_{\ell^{-1}}^{\infty} \mathcal{E}(k) d k} \equiv k_{n}^{2 n}
$$

The a priori constraints on $\mathcal{E}(k)$ are simply

$$
U^{2}=\int_{\ell^{-1}}^{\infty} \mathcal{E}(k) d k \quad \epsilon=\int_{\ell^{-1}}^{\infty} \nu k^{2} \mathcal{E}(k) d k .
$$

Suppose, in addition, that $\mathcal{E}(k)$ displays an "inertial range" in the sense that it scales with a power of $k$ up to an effective cut-off wavenumber $k_{c}$. For simplicity, let us write

$$
\mathcal{E}(k)=\left\{\begin{array}{cr}
A k^{-q}, & \ell^{-1} \leq k \leq k_{c} \\
0, & k \geq k_{c}
\end{array}\right.
$$

with $1<q<3$ and assuming that $k_{c}$ diverges as $\nu \rightarrow 0$ while $U^{2}$ and $\epsilon$ remain finite. Assuming that $A$ depends only upon the energy flux $\epsilon$ and the outer length scale $\ell$ (necessary if $q$ strays from $5 / 3$ ) we have the asymptotic relations

$$
\epsilon \sim \frac{U^{3}}{\ell} \quad \text { and } \quad \ell k_{c} \sim\left(\frac{\epsilon}{\nu^{3}}\right)^{\frac{1}{9-3 q}} \ell^{\frac{4}{9-3 q}} \sim R e^{\frac{1}{3-q}}
$$

Then the moments of the spectrum $\left\langle\kappa_{n}\right\rangle$ would satisfy

$$
\ell k_{n} \sim\left(\ell k_{c}\right)^{1-\frac{q-1}{2 n}} \sim R e^{\frac{1}{3-q}-\frac{1}{2 n}\left(\frac{q-1}{3-q}\right)} .
$$


Hence the exponents in the bounds in (65) and (70) are consistent with this spectrum only if $q \leq 8 / 3$.

Interestingly, a $k^{-8 / 3}$ energy spectrum is the borderline steepness capable of sustaining an energy cascade, as shown by Sulem and Frisch [8]. Moreover, within energy casacade models that include spatial intermittency, the $k^{-8 / 3}$ spectrum corresponds to the extreme limit where the energy dissipation is concentrated on sets of dimension zero (i.e. points) in space $[9,10]$. This view, in turn, provides some physical setting in which to interpret the result of Caffarelli, Kohn \& Nirenberg [16] on the dimension of the singularity set for weak solutions of the Navier-Stokes equations. We recall also, as pointed out by Sulem and Frisch [8], that the $k^{-8 / 3}$ spectrum follows from a Kolmogorovlike scaling argument if the spectral energy density per unit mass is replaced by the total energy spectrum and the energy flux per unit mass $\epsilon$ is replaced by the total energy dissipation rate over the entire volume (necessitating the restriction to finite volume, finite energy turbulence). So the fact is that the rigorous bounds on the moments for weak solutions derived here agree with the extreme scenario for the Fourier spectrum wavenumber scaling.

Another connection between the notion of intermittency and the methods and results described here may be established if we make some further assumptions about the flows under consideration. The opposite extreme to the fully intermittent picture described above, where the energy dissipation is concentrated on points of zero dimension in space, is the situation where the dissipation takes place uniformly in the entire three-dimensional space. The technical implementation of such an assumption is to suppose that the magnitude of the velocity gradients in the flow do not fluctuate too much in space; that is, to presume that

$$
\|\nabla \boldsymbol{u}\|_{\infty} \sim L^{-3 / 2}\|\nabla \boldsymbol{u}\|_{2} \quad \text { as } \quad R e \rightarrow \infty .
$$

As shown in [5], this assumption implies the high Reynolds number estimates

$$
\left\langle\kappa_{n, 1}^{2}\right\rangle \leq c_{n} L^{-2} R e^{3 / 2}
$$

We may re-inject this quantity into a similar calculation as that carried out in the proof of Theorem 1 to write

$$
\left\langle\kappa_{n}^{2}\right\rangle=\left\langle\kappa_{n, 1}^{\frac{2(n-1)}{n}}\left(\kappa_{1,0}^{2}\right)^{\frac{1}{n}}\right\rangle \leq\left\langle\kappa_{n, 1}^{2}\right\rangle^{\frac{(n-1)}{n}}\left(\kappa_{1,0}^{2}\right\rangle^{\frac{1}{n}} .
$$

Hence, using Lemma 1, we deduce

$$
\ell^{2 n}\left\langle\kappa_{n}^{2}\right\rangle^{n} \leq c_{n} R e^{\frac{3 n}{2}-\frac{1}{2}+2 \delta}
$$


and so

$$
\ell\left\langle\kappa_{n}\right\rangle \leq c_{n} R e^{\frac{3}{4}-\frac{1}{4 n}+\frac{\delta}{n}} .
$$

Comparing with exponents in (75), for the moments resulting from the assumed scaling-with-cut-off spectrum in (73), we see that consistency is achieved with $q \leq 5 / 3$. That is, the technical assumption of a total lack of intermittency leads to rigorous bounds on the moments of the spectrum that are saturated in Kolmogorov's classical scaling theory for homogeneous, isotropic turbulence.

This result may be quantitatively generalized to interpolate between a total lack of intermittency and a total lack of extra assumptions. We may invoke an intermediate intermittency hypothesis

$$
\|\nabla \boldsymbol{u}\|_{\infty} \approx L^{-3 / m}\|\nabla \boldsymbol{u}\|_{m} \quad 2 \leq m \leq \infty
$$

to obtain the following

Theorem 2 Under the partial intermittency suppression assumption (81)

$$
\ell\left\langle\kappa_{n}\right\rangle \leq c\left(\frac{L}{\ell}\right)^{\frac{(3 q-5)(n-1)}{n(9-3 q)}} R e^{\frac{1}{3-q}-\frac{1}{2 n}\left(\frac{q-1}{3-q}\right)+\frac{\delta}{n}}
$$

where $q=\frac{8}{3}-\frac{2}{m}$, which lies in the range

$$
5 / 3 \leq q \leq 8 / 3
$$

Proof: Firstly we use the Gagliardo-Nirenberg inequality

$$
\|A\|_{m} \leq c\left\|\nabla^{n-1} A\right\|_{2}^{a}\|A\|_{2}^{1-a}
$$

for which $a=\frac{3}{n-1}\left(\frac{1}{2}-\frac{1}{m}\right)$ with $m \geq 2$. Using (84), we can write

$$
\|\nabla \boldsymbol{u}\|_{m} \leq c \kappa_{n, 1}^{\frac{3 m-6}{2 m}} F_{1}^{1 / 2}
$$

There is an alternative version of the ladder for $F_{n}$ given in (40) of the form [5]

$$
\frac{1}{2} \dot{F}_{n} \leq-\nu F_{n+1}+\left(c_{n}\|\nabla \boldsymbol{u}\|_{\infty}+\frac{1}{g \tau^{2}}\right) F_{n} .
$$


Dividing through by $F_{n}$, time averaging and ignoring small order forcing terms, gives

$$
\left\langle\kappa_{n, 1}^{2}\right\rangle \leq\left\langle\kappa_{n+1, n}^{2}\right\rangle \leq c \nu^{-1}\left\langle\|\nabla \boldsymbol{u}\|_{\infty}\right\rangle
$$

so if we use (81) we have, for $2 \leq m \leq 6$

$$
\left\langle\kappa_{n, 1}^{2}\right\rangle \leq c \nu^{-1} L^{-3 / m}\left\langle\kappa_{n, 1}^{\frac{3 m-6}{2 m}} F_{1}^{1 / 2}\right\rangle \leq c \nu^{-1} L^{-3 / m}\left\langle\kappa_{n, 1}^{2}\right\rangle^{\frac{3 m-6}{4 m}}\left\langle F_{1}\right\rangle^{1 / 2} .
$$

This means that

$$
\ell\left\langle\kappa_{n, 1}\right\rangle \leq c\left(\frac{L}{\ell}\right)^{\frac{3(m-2)}{m+6}} R e^{\frac{3 m}{m+6}}
$$

To prove this for $m>6$ one must resort to the dynamic equation for $\kappa_{n, 1}$, dividing through by $2-\frac{6}{m}$ and then time averaging. This produces the same estimate as in (89). Using the inequality (64), the estimate in (89) can be converted into

$$
\ell\left\langle\kappa_{n, 0}\right\rangle \leq c\left(\frac{L}{\ell}\right)^{\frac{3(n-1)(m-2)}{n(m+6)}} R e^{\frac{6 m n-m(5-2 \delta)+6(1+2 \delta)}{2 n(m+6)}} .
$$

Upon choosing $q$ as

$$
q=\frac{8}{3}-\frac{2}{m}
$$

then inequality (90) becomes (82).

Acknowledgements: The authors thank Prof. Pierre-Louis Sulem and an anonymous referee for helpful remarks that have gone a long way to improve the manuscript. Various stages of this work were performed respectively at the Isaac Newton Institute program on Turbulence in 1999, the Hydrodynamic Turbulence program at ITP Santa Barbara in April 2000 and RIMS, University of Kyoto during the period 1st July - 1st October 2000. JDG would like to warmly thank RIMS for the award of a Visiting Professorship during that period and his host, Koji Ohkitani, for helpful discussions on this problem. CRD acknowledges support for this research from the US National Science Foundation. 


\section{References}

[1] J. Leray, Essai sur le mouvement d'un liquide visquex emplissant l'espace, 1934, Acta Math., 63, 193-248.

[2] Constantin P. \& Foias C., Navier-Stokes Equations, The University of Chicago Press, (Chicago, 1988).

[3] R. Temam, Infinite Dimensional Dynamical Systems in Mechanics and Physics, volume 68 of Applied Mathematical Sciences. Springer-Verlag, (New York, 1988).

[4] Foias C., Manley O., Rosa R. \& Temam R., Navier-Stokes equations and Turbulence, Cambridge University Press, (Cambridge, 2001).

[5] Doering C. R. \& Gibbon J. D., Applied analysis of the Navier-Stokes equations, Cambridge University Press, (Cambridge, 1995).

[6] Doering C. R. \& Constantin P., Energy dissipation in shear driven turbulence, 1992, Phys. Rev. Lett. 69, 1648-1651.

[7] Doering C. R. \& Foias C., Energy dissipation in body-forced turbulence, J. Fluid Mech. (in press 2002).

[8] Sulem P.-L. \& Frisch U., Bounds on energy flux for finite energy turbulence, 1975, J. Fluid Mech. 72, 417-424.

[9] Mandelbrot B. B., Some fractal aspects of turbulence: intermittency, dimension, kurtosis, and the spectral exponent $5 / 3+B$, Proc. Journées Mathématiques sur la Turbulence, Orsay (ed. R. Temam), Springer, (Berlin, 1975).

[10] Frisch U., Sulem P.-L. \& Nelkin M., A simple dynamical model of intermittent fully developed turbulence, 1978, J. Fluid Mech. 87, 719-736.

[11] Frisch U., Turbulence: The legacy of A. N. Kolmogorov, Cambridge University Press, (Cambridge, 1995).

[12] Foias C., Statistical study of Navier-Stokes equations I., 1972, Rend. Sem. Mat. Univ. Padova 48, 219-348.

[13] Foias C., Statistical study of Navier-Stokes equations II., 1973, Rend. Sem. Mat. Univ. Padova 49, 9-123.

[14] Foias C. \& Prodi G., Sur les solutions statistique des équations de Navier-Stokes, 1976, Ann. Mat. Pura Appl. (4), 111, 307-330.

[15] Foias C. Guillopé C., \& Temam R., New a priori estimates for Navier-Stokes equations in Dimension 3, 1981, Comm. in Partial Diff. Equat. 6, 329-359.

[16] Caffarelli L., Kohn R. \& Nirenberg L., Partial regularity of suitable weak solutions of the Navier-Stokes equations, 1982, Comm. Pure \& Appl. Math. 35, $771-831$. 